\title{
In vivo evidence for the unique kinetics of evoked dopamine release in the patch and matrix compartments of the striatum
}

\author{
Andrea Jaquins-Gerstl ${ }^{1}$ (D) $\cdot$ Kathryn M. Nesbitt ${ }^{1} \cdot$ Adrian C. Michael $^{1}$ \\ Received: 5 February 2021 / Revised: 11 March 2021 / Accepted: 16 March 2021 / Published online: 12 April 2021 \\ (C) The Author(s) 2021
}

\begin{abstract}
The neurochemical transmitter dopamine (DA) is implicated in a number of diseases states, including Parkinson's disease, schizophrenia, and drug abuse. DA terminal fields in the dorsal striatum and core region of the nucleus accumbens in the rat brain are organized as heterogeneous domains exhibiting fast and slow kinetic of DA release. The rates of dopamine release are significantly and substantially faster in the fast domains relative to the slow domains. The striatum is composed of a mosaic of spatial compartments known as the striosomes (patches) and the matrix. Extensive literature exists on the spatial organization of the patch and matrix compartments and their functions. However, little is known about these compartments as they relate to fast and slow kinetic DA domains observed by fast scan cyclic voltammetry (FSCV). Thus, we combined high spatial resolution of FSCV with detailed immunohistochemical analysis of these architectural compartments (patch and matrix) using fluorescence microscopy. Our findings demonstrated a direct correlation between patch compartments with fast domain DA kinetics and matrix compartments to slow domain DA kinetics. We also investigated the kinetic domains in two very distinct sub-regions in the striatum, the lateral dorsal striatum (LDS) and the medial dorsal striatum (MDS). The lateral dorsal striatum as opposed to the medial dorsal striatum is mainly governed by fast kinetic DA domains. These finding are highly relevant as they may hold key promise in unraveling the fast and slow kinetic DA domains and their physiological significance.
\end{abstract}

Keywords Voltammetry $\cdot$ Dopamine $\cdot$ Patch:matrix compartments $\cdot \mu$-Opioid receptor

\section{Introduction}

Dopamine (DA) is an immensely important neurotransmitter in the central nervous system. It contributes to motor control, responses to reward, the regulation of mood and anxiety, and several other brain functions [1,2]. Pathology of DA is clearly implicated in Parkinson's disease, dystonia, schizophrenia, attention deficit hyperactivity disorder, and substance abuse [3-7]. Consequently, drugs that target DA have wide-ranging therapeutic applications as well as illicit uses. For these reasons, understanding brain DA activity per se and the actions of DA-targeting drugs is highly significant $[8,9]$. The present

Published in the Topical Collection Electrochemistry for Neurochemical Analysis with guest editors Ashley E. Ross and Alexander G. Zestos

Andrea Jaquins-Gerstl

asj19@pitt.edu

1 Department of Chemistry, Chevron Science Center, University of Pittsburgh, 219 Parkman Ave., Pittsburgh, PA 15213, USA study extends our previous findings, based on voltammetric recordings of electrically evoked DA release, showing that the dorsal striatum in the rat is organized as a patchwork of distinct kinetic domains [10,11]. We have referred to these as the fast and slow domains, due to substantial differences in the patterns and initial rates of evoked DA release. The potential significance of these domains is highlighted by previous reports that the actions of several drugs, including DA antagonists and inhibitors of the DA transporter, are domain-specific [10]. We have identified autoinhibition as a contributing factor in the differential expression of kinetics in the domains of the dorsal striatum: the slow domains are tonically autoinhibited, but the fast domains are not. Our findings are consistent with abundant literature showing that presynaptic D2 autoreceptors regulate both DA release and DA uptake [12-18].

The objective of the present study was to explore the anatomical correlates of the fast and slow domains. Specifically, we wished to test the hypothesis that a correlation exists between the fast and slow kinetic domains and the well-known organization of the striatum into its patch (striosome) and matrix compartments [18-26]. At the outset of this work, we 
tentatively anticipated that the fast domains would correspond to patches, based simply on the observation that the fast domains, like the patch compartments, are relatively sparse in the dorsal striatum where they occupy only about $15 \%$ of the tissue [27-29]. To test this hypothesis objectively, we combined high spatial resolution voltammetric mapping of the medial and lateral portions of the dorsal striatum of the rat with detailed, post-mortem immunohistochemical analysis of the electrochemical recording sites. We based the immunohistochemical analysis on the $\mu$-opioid receptor (MOR), a wellestablished marker for the patch compartment [21, 30-32].

\section{Materials and methods}

\section{Carbon fiber microelectrodes}

Borosilicate capillaries (0.58 mm I.D., $1.0 \mathrm{~mm}$ O.D., Sutter Instruments, Novato, CA) containing a carbon fiber $(7-\mu \mathrm{m}$ diameter, T650, Cytec Carbon Fibers LLC., Piedmont, SC) were pulled to a fine tip using a vertical puller (Narishige, Los Angeles, CA, USA). The tip was sealed with epoxy (Spurr Epoxy, Polysciences Inc., Warrington, PA, USA), the fiber was cut to $200 \mu \mathrm{m}$, and the capillary was back-filled with mercury for electrical contact to a nichrome wire (Goodfellow, Oakdale, PA) [33]. Microelectrodes were soaked for $1 \mathrm{~h}$ in isopropyl alcohol (Sigma Aldrich, St. Louis, MO) prior to use [34-36].

\section{Fast-scan cyclic voltammetry}

Fast-scan cyclic voltammetry (FSCV) was performed with an EI 400 potentiostat (Ensman Instruments, Bloomington, IN) and CV Tar Heels v4.3 software. The FSCV waveform started at $0 \mathrm{~V}$ vs. $\mathrm{Ag} / \mathrm{AgCl}$ and ramped linearly $(400 \mathrm{~V} / \mathrm{s})$ to $+1.0 \mathrm{~V}$, $-0.5 \mathrm{~V}$, and $0 \mathrm{~V}$ [33]. The scan frequency was $10 \mathrm{~Hz}$. DA was background-subtracted and quantified by integration of the oxidation current between 0.5 and $0.7 \mathrm{~V}$. FSCV calibration was performed using a flow cell with $\mathrm{N}_{2}$-spurged artificial cerebrospinal fluid (aCSF: $142 \mathrm{mM} \mathrm{NaCl}, 1.2 \mathrm{mM} \mathrm{CaCl}_{2}$, $2.7 \mathrm{mM} \mathrm{KCl}, 1.0 \mathrm{mM} \mathrm{MgCl}_{2}, 2.0 \mathrm{mM} \mathrm{NaH}_{2} \mathrm{PO}_{4}, \mathrm{pH} 7.40$ ) containing DA (dopamine $\mathrm{HCl}$, Sigma Aldrich, St. Louis, MO, USA).

\section{In vivo procedures}

All procedures involving animals were approved by the Institutional Animal Care and Use Committee of the University of Pittsburgh. Sprague-Dawley rats (male, 250350 g, Charles Rivers, Raleigh, NC) were anesthetized with isoflurane (induction $5 \%$, maintenance $2.5 \%$ by volume), placed in a stereotaxic frame, and wrapped in a $37^{\circ} \mathrm{C}$ heating pad (Harvard Apparatus, Holliston, MA, USA) [33]. Two microelectrodes were implanted into the striatum along with a bipolar stimulating electrode (MS303-1-untwisted, Plastics One, Roanoke, VA). One electrode was positioned over the ipsilateral medial forebrain bundle (MFB, $4.3 \mathrm{~mm}$ posterior to bregma, $1.2 \mathrm{~mm}$ lateral from bregma, and 7.2-8.5 $\mathrm{mm}$ below dura) and lowered to a final position that evoked DA release at both microelectrodes [33]. The electrical stimulus waveform was a biphasic, constant current, square-wave (frequency $60 \mathrm{~Hz}$, pulse height $250 \mu \mathrm{A}$, pulse width $2 \mathrm{~ms}$ ) supplied via an optical isolation unit (Neurolog 800, Digitimer, Herefordshire, England).

\section{Striatal mapping}

We implanted two microelectrodes into the striatum of 10 individual rats (20 electrodes total). One electrode was aimed at the MDS (1.6 mm anterior to bregma, $1.5 \mathrm{~mm}$ lateral from bregma, and $4.5 \mathrm{~mm}$ below the surface) and the other at the LDS (0.2 $\mathrm{mm}$ anterior to bregma, $3.8 \mathrm{~mm}$ lateral from bregma, and $4.5 \mathrm{~mm}$ below the surface) for each rat [33]. Evoked DA responses were recorded along a vertical track at 6 sites of each electrode: the recording sites were $200 \mu \mathrm{m}$ apart (total track length $=1.0 \mathrm{~mm}$ : total recording sites per rat $=12$ : total recording sites $=120$ ). Figure $1 \mathrm{a}$ and $\mathrm{b}$ denote the location of the MDS and LDS recording tracks [33]. Each recording site was objectively classified as fast or slow by inspection of the response to a brief, 200-ms test stimulus. As we have described before [10, 11, 37-39], only fast sites respond to this brief test stimulus (Fig. 1c, red), whereas slow sites respond only to stimuli of longer duration (Fig. 1c, blue). Following the test stimulus, two further responses were recorded at each site using a stimulus duration of $1 \mathrm{~s}$ and $3 \mathrm{~s}$ [33]. After the recordings, the electrodes were lowered a further $500 \mu \mathrm{m}$ and their final position was marked with an electrolytic lesion (35$\mathrm{V} \mathrm{AC} 10 \mathrm{~s}$ ) to assist post-mortem histological localization of the recording tracks.

\section{Data analysis}

Statistical analysis of Fig. 3 was performed by 3-way ANOVA with time (repeated measure), track, and depth as factors: time. Statistical analysis was performed with IBM SPSS software version 22 .

\section{Tissue fixation and processing}

Tissue fixation, processing, immunochemistry, and fluorescence microscopy followed our published procedures [33, $40,41]$. After the in vivo recording session, rats were perfused with PBS and paraformaldehyde. The tissue was soaked in $2 \%$ paraformaldehyde for $2 \mathrm{~h}$ followed by $30 \%$ sucrose overnight, frozen in liquid nitrogen-cooled 2-methylbutane, stored at $-80{ }^{\circ} \mathrm{C}$, and sliced vertically in a cryostat into $30-\mu \mathrm{m}$-thick 

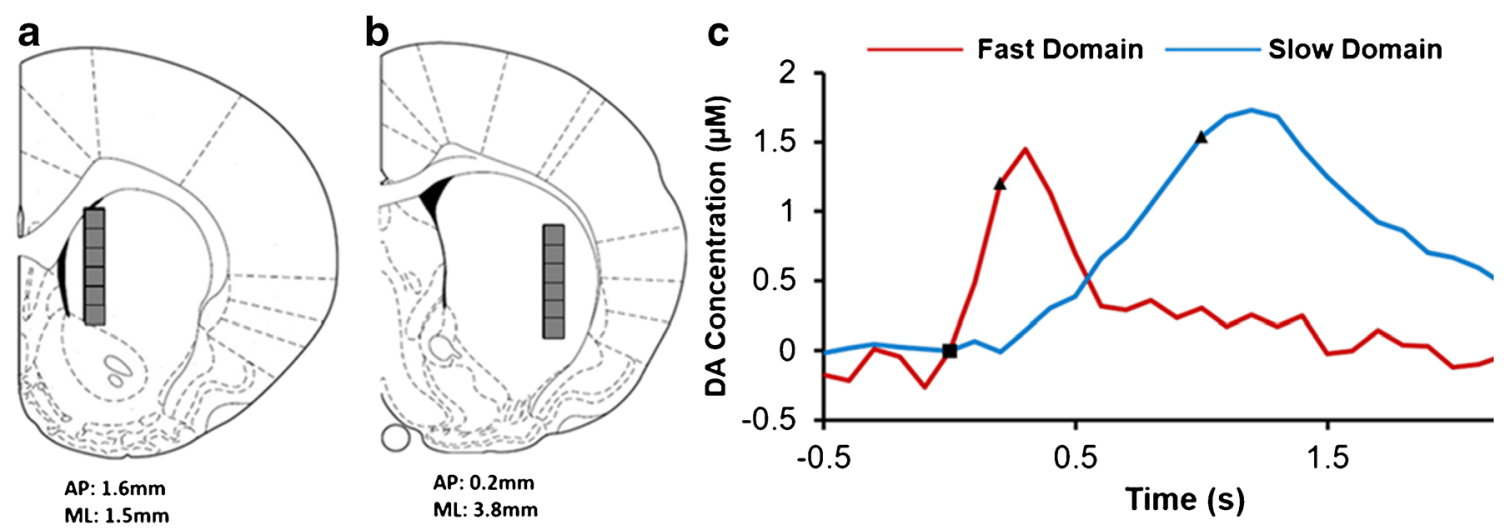

Fig. 1 Schematic of the locations of the recording electrode tracks in the a MDS and $\mathbf{b}$ LDS. $\mathbf{c}$ Representative examples of fast (red) and slow (blue) evoked DA responses in the rat striatum: the symbols show when each stimulus starts (square) and stops (triangles)

sections. The sections were placed into cryoprotection solution and stored at $20^{\circ} \mathrm{C}$ until immunolabeled.

\section{Immunofluorescence and fluorescence microscopy}

Immunohistochemistry was performed using tyramide signal amplification (TSA) on free-floating sections to determine the distributional patterns of the $\mu$-opioid receptor, MOR [33, 42-45]. Tissue sections were labeled with the primary antibody (rabbit polyclonal MOR antibody, 1:100,000; Millipore) in a $20 \%$ goat serum blocking solution at room temperature for $48 \mathrm{~h}$. The secondary antibody was IgG labeled with fluorescein $(1: 1000$; Perkin Elmer, Shelton, CT). Fluorescence microscopy (Olympus BX61, Olympus; Melville, NY) used a $\times 1.25$ or $\times 10$ objective and wavelength matched filter sets (Chroma Technology; Rockingham, VT). Images were analyzed and quantified with Metamorph/Fluor 7.1 (Universal Imaging Corporation; Molecular Devices), NIS Elements AR (Nikon Corporation; Tokyo, Japan), and OriginPro.

\section{Results}

\section{Domain classification}

Due in part to their high spatial and temporal resolution, carbon fiber microelectrodes have proven valuable in the study of central DA systems. Measurements of extracellular DA in the rat striatum during electrical stimulation of nigrostriatal DA axons in the MFB have revealed that the striatal DA terminal field forms a patchwork of distinct fast and slow kinetic domains $[11,38]$. Furthermore, some DA-targeting drugs, including DA antagonists and inhibitors of the DA transporter, exhibit domain-specific actions $[10,11,46]$. In prior work [11, 38], we established an objective procedure to classify recording sites as fast or slow type based on the response to a brief test stimulus of $200 \mathrm{~ms}$ in duration (at $60 \mathrm{~Hz}$, the test stimulus consists of 12 stimulus pulses). Only fast sites respond to this test stimulus (Fig. 2a, red). Slow sites do not respond to the test stimulus but respond robustly to longer stimuli (Fig. 2a, blue). Figure 2a reports fast and slow responses recorded simultaneously at two carbon fiber microelectrodes implanted side-by-side in a single rat during the same two stimuli. This reinforces the conclusion that the recording site determines whether the response is fast or slow type. The overall amplitude of the 3-s response from the slow site in Fig. 2a happens to be larger than that from the fast site: this emphasizes that the classification of a site as fast or slow relies on the response to the test stimulus rather than the overall response amplitude.

The microelectrodes used in this work had an active length of $200 \mu \mathrm{m}$ (see "Materials and methods"). Therefore, each movement of the microelectrode by $200 \mu \mathrm{m}$ along its track is presumed to establish a new recording site. Figure $2 \mathrm{~b}$ reports a set of six responses recorded along a single microelectrode track. Based on their responses to the test stimulus, three of the responses are from fast sites (red) and three from slow sites (blue): no two responses are the same (see also Supplementary Fig. S2). Figure 2c reports representative fast and slow responses from all the nominal recording sites selected for this study (Fig. 2c combines responses from different microelectrodes and different rats). Collectively, these data confirm that each nominal recording site is unique and that none is exclusively fast or exclusively slow.

\section{Domains in the lateral and medial dorsal striatum}

The overall amplitude of 1-s responses from fast sites is larger than from slow sites in both the MDS and LDS (Fig. 3a). In addition, overall response amplitudes from the MDS were significantly larger than from the LDS (details of the statistical analysis are reported in the figure legend). Even though the MDS produced the larger overall response amplitudes, the MDS produced fewer fast responses than the LDS (28\% and $53 \%$, respectively: Fig. 3b). This is consistent with the point made above that the classification of fast and slow sites rests on the test stimulus rather than the overall response amplitude. 
a
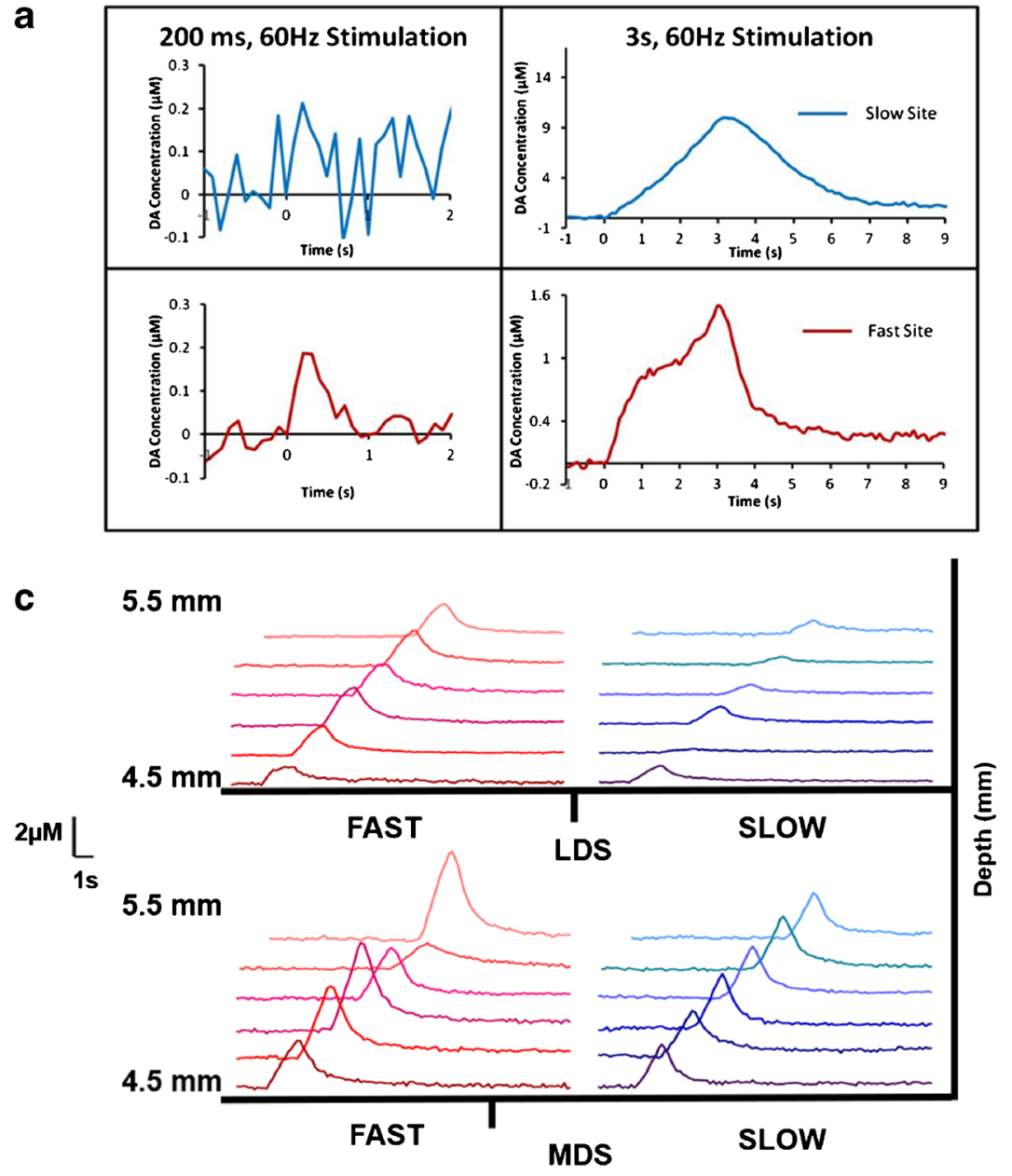

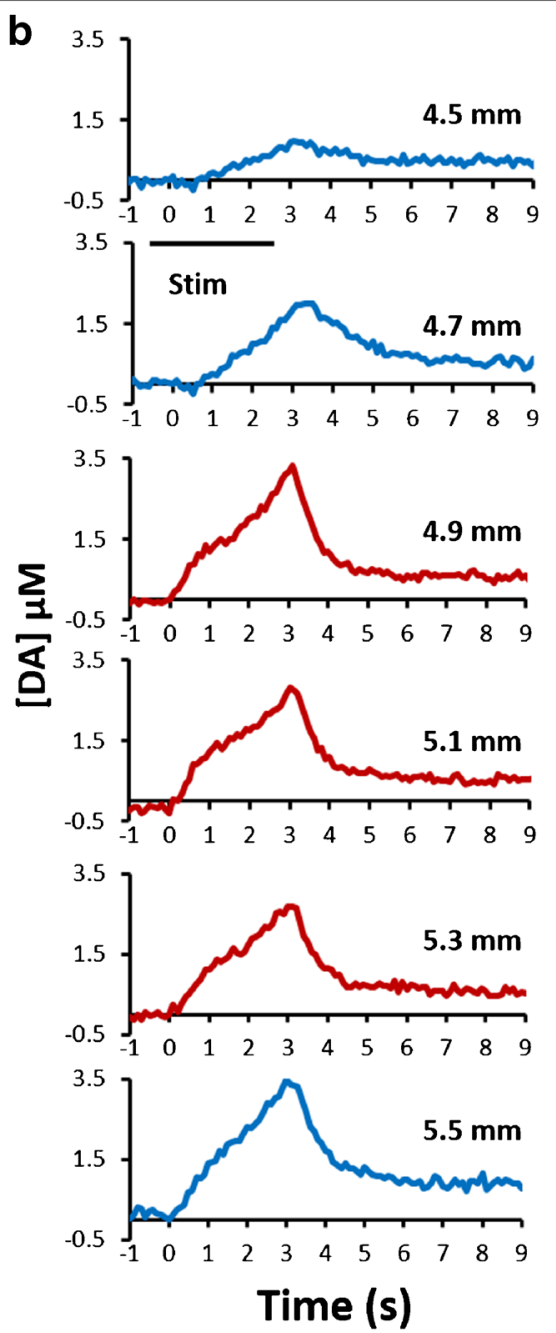

Fig. 2 a Fast (red) and slow (blue) evoked DA responses recorded simultaneously with side-by-side carbon fiber microelectrodes in a single rat during the same electrical stimuli. b Responses from a single microelectrode track exhibiting three fast (red) and three slow (blue) sites. c

Collectively, there are significant differences between the MDS and LDS in both the overall response amplitudes and the incidence of fast and slow sites.

\section{The correlation between domains and compartments}

We inspected individual recording sites by means of MOR immunofluorescence to test the hypothesis that a correlation exists between DA's fast and slow kinetic domains and the patch and matrix compartments of the striatum. The MOR is a well-established histochemical marker for striosomes (patches). Spots of heightened MOR immunoreactivity are scattered throughout the striatal regions of all the tissue sections examined (green, Fig. 4a). A subcallosal streak of intense MOR reactivity bordering the striatum is also present, in agreement with prior studies based on $\left[{ }^{3} \mathrm{H}\right]$-naloxone
Representative fast and slow response from all the nominal recording locations selected for this study: none of the nominal recording sites was exclusively fast or exclusively slow

binding $[47,48]$. MOR immunoreactivity was generally absent in other regions of the tissue, including cortical regions. Many of the less intense spots of MOR immunoreactivity are attributable to presumed non-specific labeling of myelinated axon bundles observable as dark spots in the differential interference contrast (DIC) images of the same tissue section (Fig. 4b). Therefore, the DIC and immunofluorescence images were superimposed, a fluorescence threshold intensity was set to eliminate the less intense spots of fluorescence associated with axon bundles, and spots of more intense fluorescence were outlined in the software (Fig. 4c). The computer-outlined spots are presumed to be the striatal patches (striosomes) embedded within the relatively MOR-free striatal matrix.

Based on past experience, we anticipated that the microelectrode tracks would not be visible under light microscopy 

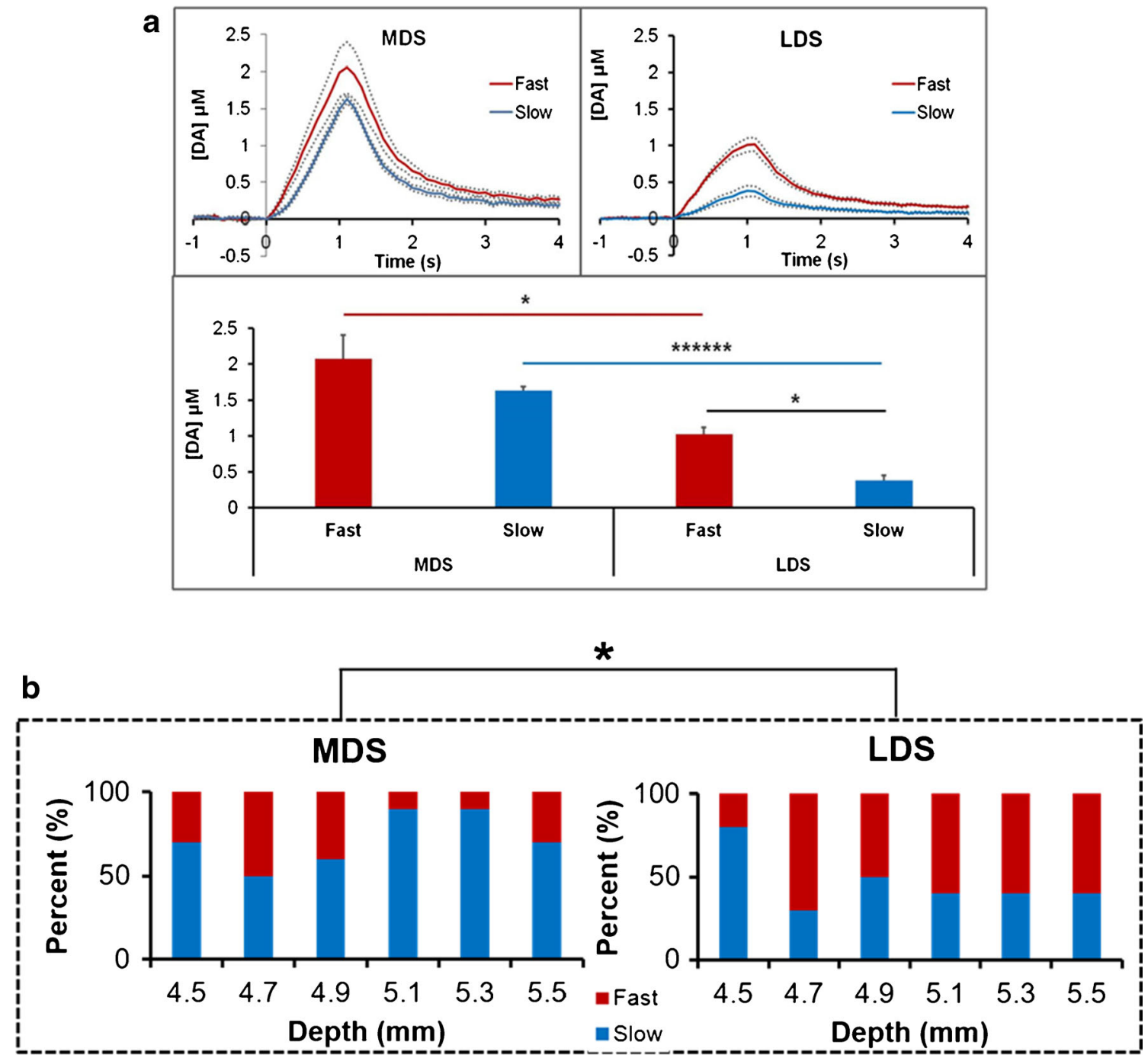

Fig. 3 a Top: The overall average of fast (red) and slow (blue) evoked DA responses in the MDS and LDS (dotted lines show standard deviations). Bottom: A summary of the overall response amplitudes. Statistical analysis was by 2 -way ANOVA with repeated measures with location (medial, lateral) and response type (repeated measure, fast, slow) as factors. Both location $\mathrm{F}_{(1,10)}=42.9, p=0.000065$, and response $\mathrm{F}_{(1,10)}=$ $8.65, p=0.015$, were significant factors. Interaction between factors

[10], so we marked the bottom of each track with an electrolytic lesion. Such lesions are visible by DIC (Fig. 4b, red circle). The locations of the recording sites were estimated by measuring upwards from the lesion. Unfortunately, some of the lesions were too large to allow a precise determination of the recording site locations. Therefore, the analysis that follows is based on the subset of electrode tracks with sufficiently small lesions, which we defined as those appearing in fewer than three adjacent tissue sections (less than $90 \mu \mathrm{m}$ ). We analyzed 7 tracks in all, 2 in the LDS and 5 in MDS, with a total of 42 recording sites. The estimated locations of the recording sites along a representative track are superimposed on the outline drawing of the patches (Fig. 4c), with fast sites drawn in red and slow sites drawn in white. was not significant. Post hoc pairwise comparisons with Bonferroni correction revealed a significant difference between fast and slow responses in the LDS only $(* p<0.05, * * * * * * p<0.0000005)$. b The number of fast (red) and slow (blue) responses at each nominal recording site. The average number of fast responses per site in the LDS is significantly larger than that in the MDS (t-test, $\left.{ }^{*} p<.05\right)$

Figure 5 summarizes the correlation between fast and slow sites and the patch and matrix compartments. Figure 5a explains the analysis of an individual track. The fast and slow recording sites are color coded in red and blue, respectively: patches that coincide with fast sites are outlined in red (Fig. 4c also shows three red patches), while a patch that coincides with a slow site is outlined in blue. Figure $5 \mathrm{~b}$ reports the 3-s responses from each site along the track in Fig. 5a: the response from the single fast site is coded in red and responses from the 5 slow sites are coded in blue; responses from sites that coincide with the patch compartment are outlined in green and from the matrix compartment are outlined in black. In this case, the single fast site coincides with the patch compartment, three slow sites coincide with the matrix compartment, and 

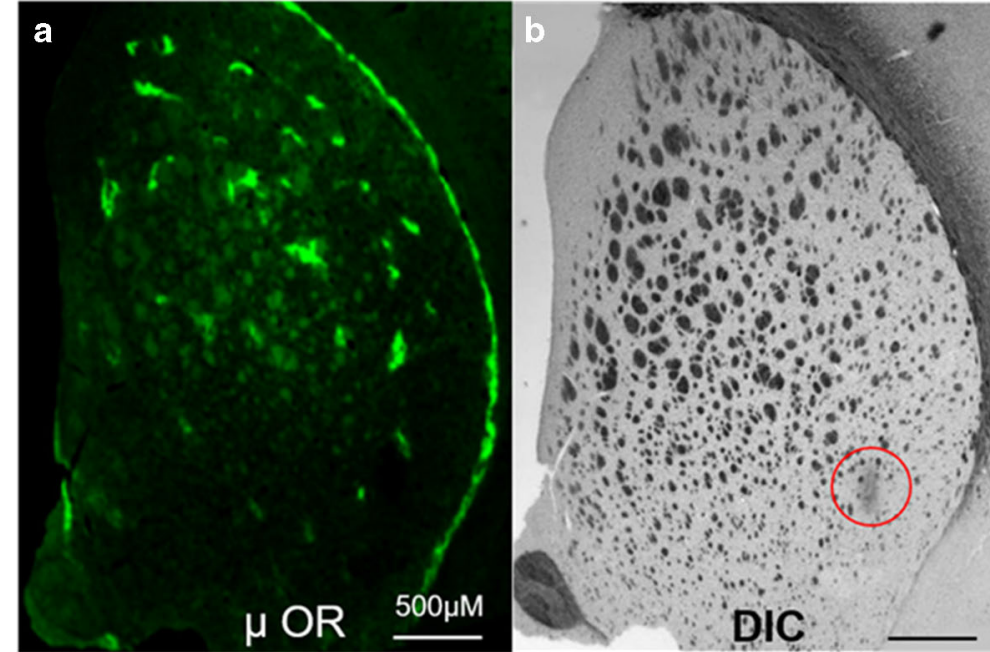

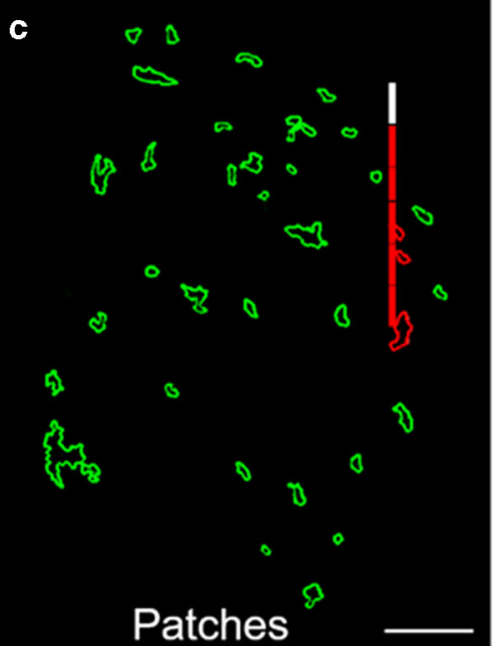

drawing of the computer-identified MOR-positive patches and a scale drawing of an electrode track with fast sites in red and a slow site in white. Scale bars are $500 \mu \mathrm{m}$

the matrix compartment. However, a few fast sites (13\%) coincide with the matrix and a few slow sites $(7 \%)$ with patches: this is discussed further in the next section.

Figure $5 \mathrm{~d}$ reports the proportion of recording sites in the MDS and LDS identified as corresponding to the patch partment and slow sites predominantly (93\%) coincide with

a

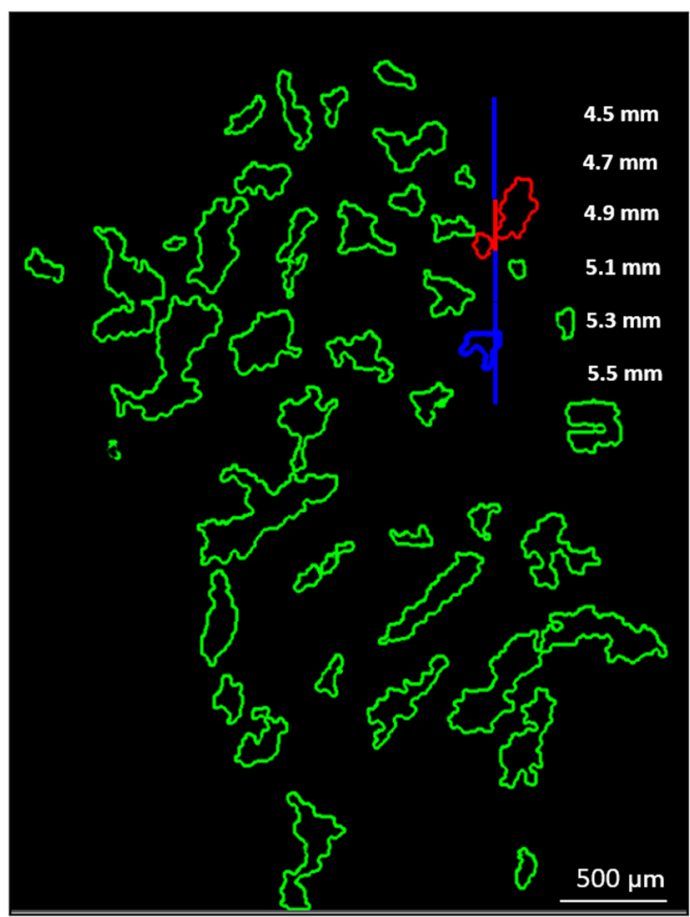

b

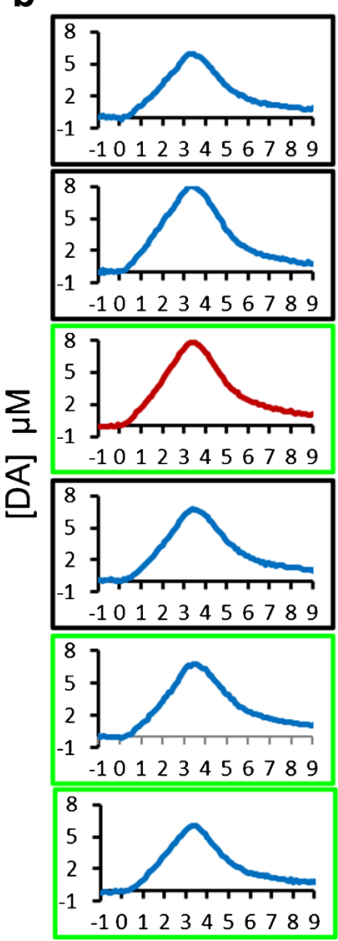

C

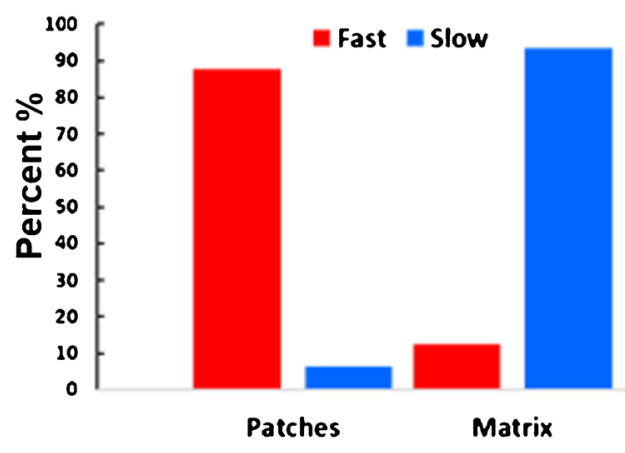

d

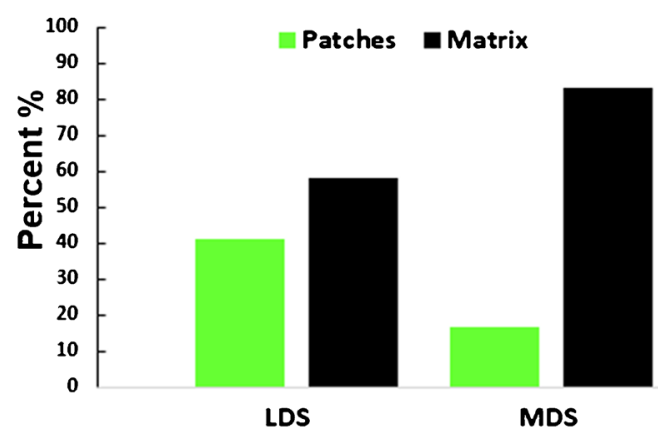

Fig. 5 a Drawing of an electrode track superimposed on an outline drawing of MOR-positive patches in a striatal tissue section. The single fast site is red and the five slow sites are blue. Patches that coincide with the fast site are in red and a patch that coincides with two slows site is in blue. Scale bar is $500 \mu \mathrm{m}$. b Evoked DA responses from each recording site along the track displayed in panel a with the fast response in red and the five slow responses in blue. Responses from sites in the patch compartment are outlined in green and responses from sites in the matrix compartment are outlined in black. c Correlation of fast:slow sites with patch:matrix compartments, expressed as a percent. d Proportion of patch and matrix sites within the LDS and MDS, expressed as a percent 
(green) and matrix (black) compartments. Although Fig. 5d is based on a subset of the recording sites, more recording sites in the LDS coincide with patches (40\%) than in the MDS $(17 \%)$. Thus, the results summarized in Figs. $3 \mathrm{~b}$ and $5 \mathrm{~d}$ together show that we encountered more fast sites and patches in the LDS than in the MDS.

\section{Proximity of fast sites to patches}

The correlation between domains and compartments reported in Figs. 4 and 5 is based on a count of fast sites that coincide with a patch. However, a few fast sites (13\%) did not coincide with a patch (Fig. 5b). Therefore, we wished to examine these sites in closer detail. Figure 6a is a surface intensity plot of the MOR immunofluorescence near a track in the LDS with two fast sites that did not coincide with a patch. Circles with diameters of 100 , 200 , and $300 \mu \mathrm{m}$ are centered on several patches in the vicinity of the track. As in the case of this representative track, all fast sites in this study coincide with at least one of these circles. A similar analysis along a track in the MDS (Fig. 6b) that produced only slow sites shows that none of the recording sites coincides with any circles. Although this analysis does not account for the few slow sites that coincide with patches (Fig. 5c), we conclude that all the fast-recording sites in this study either coincide with or are proximal to striatal patches.

\section{Discussion}

The findings of this study support the conclusion that the patchwork of fast and slow kinetic domains within the striatal DA terminal field correspond, respectively, to the patch and matrix striatal compartments. Across the LDS and MDS, $87 \%$ of fast sites coincided with a patch (Fig. 5) and the remaining 13\% were proximal to a patch, i.e., located within $300 \mu \mathrm{m}$ of a patch (Fig. 6). Meanwhile, $93 \%$ of slow sites coincided with the matrix and $7 \%$ coincided with a patch. Thus, fast and slow sites are predominantly associated with the patch and matrix compartments, respectively. In general terms, this conclusion supports the concept that the kinetics of DA release, diffusion, and clearance are finetuned to the local neurocircuitry, functional significance, and anatomical organization of the striatal brain region $[49,50]$.

The patch:matrix architecture of the striatum and its role in brain function and disease are well-known. For example, Graybiel and colleagues have examined how imbalances in patch and matrix properties, such as cell densities and gene expression, contribute to numerous diseases, such as Parkinson's, Huntington's, dystonia, and addiction [22, 51-56]. Likewise, the medial and lateral regions of the dorsal striatum, which are associated with associative and sensorimotor functions, respectively [51, 57-61], are implicated in obsessive-compulsive disorders. While the findings of the present study add to the body of information regarding the distinct properties of the patch and matrix compartments, the functional significance of the distinct DA kinetics between compartments remains to be elucidated. Further studies will be required to establish why, for example, the patches exhibit faster DA kinetics than the matrix.

It has long been conjectured that the relatively rare "hot-spots" of evoked DA release within the striatum might correspond to striatal patches, which represent the smaller and relatively sparse striatal compartment. Evidence suggests that the patch and matrix compartments receive specific projections from midbrain DA neurons [1, 62-64]. For this reason, we have wondered if the distinct fast and slow profiles are attributable to different types of midbrain DA neurons. Our studies, however, do not support this idea. Our work shows that the fast and slow profiles are readily predicted by a single mathematical model of evoked DA release kinetics, with the fast and slow responses requiring only different
Fig. 6 A color-coded surface intensity plot of MOR immunofluorescence in the vicinity of a microelectrode track in the a LDS and $\mathbf{b}$ MDS: patches and the subcallosal streak appear as spots of yellow and orange: the matrix appears mostly in green and blue (color scale is at the top of the figure). Circles with diameters of 100,200 , and $300 \mu \mathrm{m}$ are centered on the patches. All fast sites (red) coincide with a circle. In this case, none of the slow sites coincides with any circles

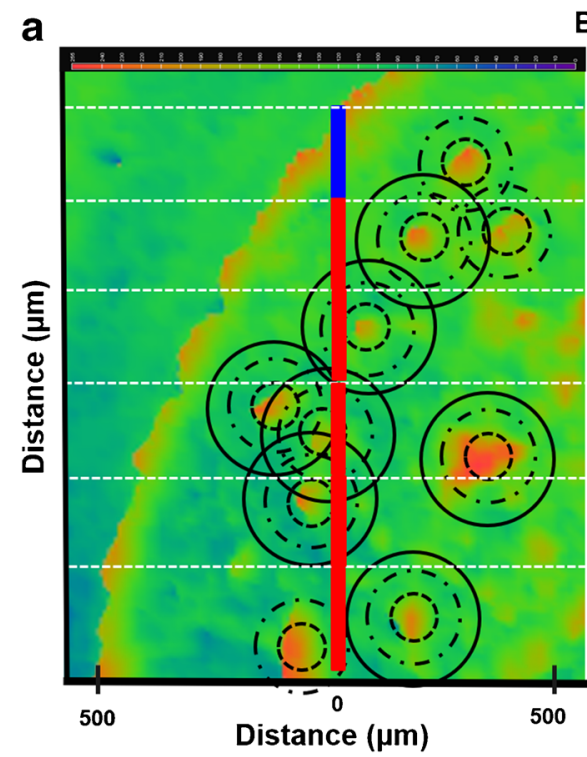

Below Dura

$4.5 \mathrm{~mm}$

$4.7 \mathrm{~mm}$

$4.9 \mathrm{~mm}$

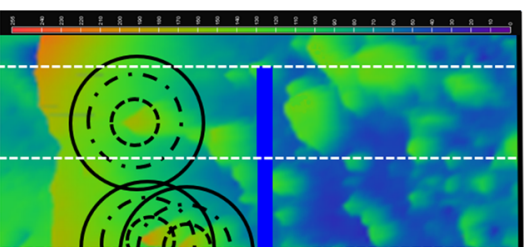

$5.1 \mathrm{~mm}$

$5.3 \mathrm{~mm}$
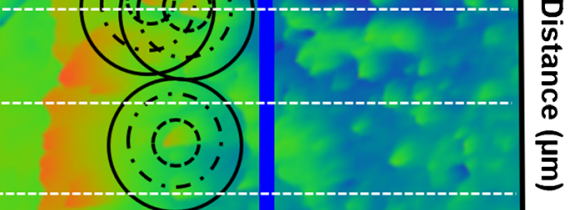

$5.5 \mathrm{~mm}$

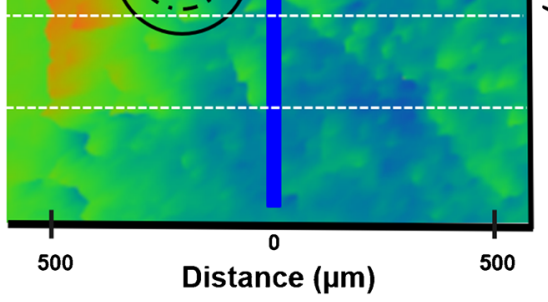


values of the various rate parameters [39, 65-69]. This modeling work, therefore, does not illuminate any inherent difference in the mechanisms of DA release, diffusion, and clearance that produce fast and slow responses. In light of this, we speculate that the fast and slow kinetics of evoked DA release arise from the local neurochemical environment of the DA terminals themselves. It is well-known, for example, that DA release is regulated at the pre-synaptic level by DA autoreceptors [37, 70] and heteroreceptors [1, 71-74].

We did encounter a few "mismatches" during our study: $13 \%$ of fast sites coincided with the matrix and $7 \%$ of slow sites coincided with a patch. Several factors could potentially contribute to these few mismatches. One possible factor is methodological limitations. Histological verification of carbon fiber microelectrode tracks is challenging because they are so small. During this study, we used a lesion as a histological reference mark. In several cases, however, the lesion was too large to provide a precise point of reference. Even when the lesion was sufficiently compact, accurate verification of the recording sites requires precise alignment of the tissue sections with the stereotaxic plane of the electrode tracks, which is also quite challenging. Hence, uncertainty in the location of the recording sites could have contributed to the few mismatches observed during this work.

Furthermore, there are some limitations inherent in immunohistochemical tissue analysis. First, the tissue was sectioned in the coronal plane of the electrode tracks: thus, it is possible that nearby patches in adjacent tissue sections were missed. Second, the presumed non-specific labeling of myelinated axon bundles made it necessary to threshold the images in order to identify the patches: it is possible that the thresholding procedure concealed the full extent of the patches.

We must consider the possibility that DA's fast domains are "correlated with" but do not exactly correspond to patches, i.e., that the extent of the fast domains and the patch are not identical. This seems plausible, given that MORs are expressed by striatal neurons rather than DA terminals. Such a possibility was raised previously by Cragg $[64,68]$ who, by examining evoked DA release in an ex vivo striatal slice preparation, found evidence for a "peristriosomal" region, i.e., a region of unique evoked DA release in regions just surrounding striatal patches. It is possible that the fast sites we identified as "proximal" to a patch correspond to these peristriosomal regions.

As just mentioned, the conclusion that the patch and matrix striatal compartments exhibit distinct kinetics of evoked DA release is supported by prior studies in ex vivo slice preparations. A major advantage of ex vivo preparations is the greater reliability of correlating recording sites with the patch or matrix compartments. Brimblecomb and Cragg deposited a marker at the recording sites and used MOR immunofluorescence to identify patches in the same slice [64]. Salinas et al. employed a mouse strain expressing a GFP-fusion of the striosome-specific protein, Nr4a1 [69].

Nevertheless, there are interesting differences between the findings from the in vivo and ex vivo preparations. The most dramatic difference is that the ex vivo preparations do not exhibit the slow-type release dynamics observed in vivo. In the ex vivo studies, single electrical stimulus pulses evoked robust DA release at all sites, with larger responses observed in the matrix compared to the patch. This contrast is undoubtedly due to inherent differences between the in vivo and ex vivo preparations. For instance, Moquin and Michael suggested that slow-type evoked DA responses are attributable to an autoinhibitory tone of basal extracellular dopamine acting on presynaptic DA autoreceptors [11]. It appears possible that the ex vivo slice is unable to maintain this tone, perhaps due to a washout of extracellular DA, and thus does not exhibit the slow-type evoked DA profile. This is consistent with the observations of Moquin and Michael that the D2 receptor antagonist, raclopride, converts slow sites into fast sites of evoked DA release, whereas Salinas et al. report that autoinhibition does not contribute to patch:matrix differences in evoked release [69].

Another methodological difference between in vivo and ex vivo preparations might be important to consider. In our in vivo studies, the electrical stimulus was applied to ascending DA axons in the MFB, whereas the stimulus in the ex vivo preparations was applied locally and directly to the terminal field. Thus, it is likely that different neuronal circuit elements are stimulated in the two preparations. For example, Cragg and colleagues have demonstrated that cholinergic interneurons within the striatum powerfully modulate DA [71]; however, striatal interneurons would presumably not be directly activated by electrical stimulation of the midbrain. So, it might be the case the slow-type evoked DA release is specific to the axonal pathway stimulation of the in vivo preparation.

Overall, however, it is remarkable that the main conclusion, that the patch and matrix compartments exhibit distinct kinetics of evoked DA release, is mutually reinforced by studies of both the in vivo and the ex vivo preparations. This adds substantially to the robustness of the main conclusion of these collective works that the dynamics of DA transmission, and the mechanisms of actions of several DA-targeting drugs are unique to the patch and matrix compartments of the striatum.

Supplementary Information The online version contains supplementary material available at https://doi.org/10.1007/s00216-021-03300-z.

Funding This work was supported by grants from The National Institutes of Health (MH075989, NS086107).

\section{Declarations}

Ethics approval Procedures involving animals were approved by the Institutional Animal Care and Use Committee of the University of Pittsburgh.

Conflict of interest The authors declare no competing interests. 
Open Access This article is licensed under a Creative Commons Attribution 4.0 International License, which permits use, sharing, adaptation, distribution and reproduction in any medium or format, as long as you give appropriate credit to the original author(s) and the source, provide a link to the Creative Commons licence, and indicate if changes were made. The images or other third party material in this article are included in the article's Creative Commons licence, unless indicated otherwise in a credit line to the material. If material is not included in the article's Creative Commons licence and your intended use is not permitted by statutory regulation or exceeds the permitted use, you will need to obtain permission directly from the copyright holder. To view a copy of this licence, visit http://creativecommons.org/licenses/by/4.0/.

\section{References}

1. Rice ME, Patel JC, Cragg SJ. Dopamine release in the basal ganglia. Neuroscience. 2011;198:112-37.

2. Schultz W. Multiple dopamine functions at different time courses. Annu Rev Neurosci. 2007;30:259-88.

3. Bao L, Patel JC, Walker RH, Shashidharan P, Rice ME. Dysregulation of striatal dopamine release in a mouse model of dystonia. J Neurochem. 2010;114(6):1781-91.

4. de la Fuente-Fernández R, Schulzer M, Kuramoto L, Cragg J, Ramachandiran N, Au WL, et al. Age-specific progression of nigrostriatal dysfunction in Parkinson's disease. Ann Neurol. 2011;69(5):803-10.

5. Koob GF, Bloom FE. Cellular and molecular mechanisms of drug dependence. Science. 1988;242(4879):715-23.

6. Russell VA, Sagvolden T, Johansen EB. Animal models of attention-deficit hyperactivity disorder. Behav Brain Funct. 2005;1(1):9.

7. Steiner H, Tseng KY. Handbook of basal ganglia structure and function: Academic Press; 2016.

8. Virdee K, Cumming P, Caprioli D, Jupp B, Rominger A, Aigbirhio FI, et al. Applications of positron emission tomography in animal models of neurological and neuropsychiatric disorders. Neurosci Biobehav Rev. 2012;36(4):1188-216.

9. Westerink BH, Cremers TI. Handbook of microdialysis: methods, applications and perspectives: Academic Press; 2007.

10. Mitch Taylor I, Jaquins-Gerstl A, Sesack SR, Michael AC. Domain-dependent effects of DAT inhibition in the rat dorsal striatum. J Neurochem. 2012;122(2):283-94.

11. Moquin KF, Michael AC. Tonic autoinhibition contributes to the heterogeneity of evoked dopamine release in the rat striatum. J Neurochem. 2009;110(5):1491-501.

12. Calipari ES, Huggins KN, Mathews TA, Jones SRJNi. Conserved dorsal-ventral gradient of dopamine release and uptake rate in mice, rats and rhesus macaques. Neurochem Int. 2012;61(7):98691.

13. Eblen F, Graybiel AM. Highly restricted origin of prefrontal cortical inputs to striosomes in the macaque monkey. J Neurosci. 1995;15(9):5999-6013.

14. Gulley JM, Zahniser NR. Rapid regulation of dopamine transporter function by substrates, blockers and presynaptic receptor ligands. Eur J Pharmacol. 2003;479(1-3):139-52.

15. Capper-Loup C, Canales JJ, Kadaba N, Graybiel AM. Concurrent activation of dopamine D1 and D2 receptors is required to evoke neural and behavioral phenotypes of cocaine sensitization. J Neurosci. 2002;22(14):6218-27.

16. Cragg SJ, Rice ME. DAncing past the DAT at a DA synapse. Trends Neurosci. 2004;27(5):270-7.

17. Gerfen CR, Engber TM, Mahan LC, Susel Z, Chase TN, Monsma FJ, et al. D1 and D2 dopamine receptor-regulated gene expression of striatonigral and striatopallidal neurons. Science. 1990;250(4986):1429-32.

18. Sesack SR, Aoki C, Pickel VM. Ultrastructural localization of D, receptor-like immunoreactivity in midbrain dopamine neurons and their striatal targets. J Neurosci. 1994;14(1):88-106.

19. Graybiel AM, Ragsdale CW Jr. Histochemically distinct compartments in the striatum of human, monkeys, and cat demonstrated by acetylthiocholinesterase staining. Proc Natl Acad Sci U S A. 1978;75(11):5723-6.

20. Bolam JP, Izzo PN, Graybiel AM. Cellular substrate of the histochemically defined striosome/matrix system of the caudate nucleus: a combined Golgi and immunocytochemical study in cat and ferret. Neuroscience. 1988;24(3):853-75.

21. Crittenden JR, Graybiel AM. Basal ganglia disorders associated with imbalances in the striatal striosome and matrix compartments. Front Neuroanat. 2011;5.

22. Crittenden JR, Graybiel AM. Chapter 39 - disease-associated changes in the striosome and matrix compartments of the dorsal striatum. In: Steiner H, Tseng KY, editors. Handbook of behavioral neuroscience. 24: Elsevier; 2016. p. 783-802.

23. Jimenez-Castellanos J, Graybiel AM. Subdivisions of the dopamine-containing A8-A9- A10 complex identified by their differential mesostriatal innervation of striosomes and extrastriosomal matrix. Neuroscience. 1987;23(1):223-42.

24. Langer LF, Graybiel AM. Distinct nigrostriatal projection systems innervate striosomes and matrix in the primate striatum. Brain Res. 1989;498(2):344-50.

25. Desban M, Kemel M, Glowinski J, Gauchy C. Spatial organization of patch and matrix compartments in the rat striatum. Neuroscience. 1993;57(3):661-71.

26. Holt DJ, Graybiel AM, Saper CB. Neurochemical architecture of the human striatum. J Comp Neurol. 1997;384(1):1-25.

27. Hanley JJ, Bolam JP. Synaptology of the nigrostriatal projection in relation to the compartmental organization of the neostriatum in the rat. Neuroscience. 1997;81(2):353-70.

28. Murray RC, Logan MC, Horner KA. Striatal patch compartment lesions reduce stereotypy following repeated cocaine administration. Brain Res. 1618;2015:286-98.

29. Whalley K. Patch work in the striatum. Nat Rev Neurosci. 2016;17(2):75.

30. Hirsch EC, Graybiel AM, Hersh LB, Duyckaerts C, Agid Y. Striosomes and extrastriosomal matrix contain different amounts of immunoreactive choline acetyltransferase in the human striatum. Neurosci Lett. 1989;96(2):145-50.

31. Johnston JG, Gerfen CR, Haber SN, van der Kooy D. Mechanisms of striatal pattern formation: conservation of mammalian compartmentalization. Brain Res Dev Brain Res. 1990;57(1):93-102.

32. Tremblay L, Kemel M-L, Desban M, Gauchy C, Glowinski J. Distinct presynaptic control of dopamine release in striosomal-and matrix-enriched areas of the rat striatum by selective agonists of NK1, NK2, and NK3 tachykinin receptors. Proc Natl Acad Sci U S A. 1992;89(23):11214-8.

33. Nesbitt KM. Retrodialysis of pharmacological agents mitigates tissue damage during brain microdialysis and preserves dopamine activity in surrounding tissue [Ph.D.]. Pittsburgh: University of Pittsburgh; 2015.

34. Di S, Barth DS. The functional anatomy of middle-latency auditory evoked potentials: thalamocortical connections. J Neurophysiol. 1992;68(2):425-31.

35. Barth BB, Huang H-I, Hammer GE, Shen X. Opportunities and challenges for single- unit recordings from enteric neurons in awake animals. Micromachines. 2018;9(9):428.

36. Sukov W, Barth DS. Three-dimensional analysis of spontaneous and thalamically evoked gamma oscillations in auditory cortex. J Neurophysiol. 1998;79(6):2875-84. 
37. Moquin KF, Michael AC. An inverse correlation between the apparent rate of dopamine clearance and tonic autoinhibition in subdomains of the rat striatum: a possible role of transportermediated dopamine efflux. J Neurochem. 2011;117(1):133-142.

38. Taylor IM, Ilitchev AI, Michael AC. Restricted diffusion of dopamine in the rat dorsal striatum. ACS Chem Neurosci. 2013;4(5): $870-8$.

39. Taylor IM, Nesbitt KM, Walters SH, Varner EL, Shu Z, Bartlow $\mathrm{KM}$, et al. Kinetic diversity of dopamine transmission in the dorsal striatum. J Neurochem. 2015;133(4):522-531.

40. Jaquins-Gerstl A, Michael AC. Comparison of the brain penetration injury associated with microdialysis and voltammetry. J Neurosci Methods. 2009;183(2):127-35.

41. Mitala CM, Wang Y, Borland LM, Jung M, Shand S, Watkins S, et al. Impact of microdialysis probes on vasculature and dopamine in the rat striatum: a combined fluorescence and voltammetric study. J Neurosci Methods. 2008;174(2):177-85.

42. Koizumi H, Morigaki R, Okita S, Nagahiro S, Kaji R, Nakagawa $\mathrm{M}$, et al. Response of striosomal opioid signaling to dopamine depletion in 6-hydroxydopamine-lesioned rat model of Parkinson's disease: a potential compensatory role. Front Cell Neurosci [Internet]. 2013 2013; 7:[74 p.]. Available from: http:// europepmc.org/abstract/MED/23730270.

43. Shinoda R, Saito T, Okita Y, Isogai A. Relationship between length and degree of polymerization of TEMPO-oxidized cellulose nanofibrils. Biomacromolecules. 2012;13(3):842-9.

44. Scherrer G, Imamachi N, Cao Y-Q, Contet C, Mennicken F, O'Donnell D, et al. Dissociation of the opioid receptor mechanisms that control mechanical and heat pain. Cell. 2009;137(6):1148-59.

45. Bao Y, Gao Y, Yang L, Kong X, Yu J, Hou W, et al. The mechanism of $\mu$-opioid receptor (MOR)-TRPV1 crosstalk in TRPV1 activation involves morphine anti-nociception, tolerance and dependence. Channels (Austin). 2015;9(5):235-43.

46. Wang Y, Moquin KF, Michael AC. Evidence for coupling between steady-state and dynamic extracellular dopamine concentrations in the rat striatum. J Neurochem. 2010;114(1):150-9.

47. Schnittler M, Liebmann C, Schrader U, Schulze HP, Neubert K, Repke H. [3H]naloxone as an opioid receptor label: analysis of binding site heterogeneity and use for determination of opioid affinities of casomorphin analogues. Biomed Biochim Acta. 1990;49(4):209-18.

48. Krebs M-O, Gauchy C, Desban M, Glowinski J, Kemel M. Role of dynorphin and GABA in the inhibitory regulation of NMDAinduced dopamine release in striosome-and matrix-enriched areas of the rat striatum. J Neurosci. 1994;14(4):2435-43.

49. Carr DB, Sesack SR. Projections from the rat prefrontal cortex to the ventral tegmental area: target specificity in the synaptic associations with mesoaccumbens and mesocortical neurons. J Neurosci. 2000;20(10):3864-73.

50. Sesack SR, Grace AA. Cortico-basal ganglia reward network: microcircuitry. Neuropsychopharmacology. 2010;35(1):27-47.

51. Graybiel AM. Habits, rituals, and the evaluative brain. Annu Rev Neurosci. 2008;31:359-87.

52. Graybiel AM. The basal ganglia and cognitive pattern generators. Schizophr Bull. 1997;23(3):459-69.

53. Graybiel AM, Hirsch EC, Agid Y. The nigrostriatal system in Parkinson's disease. Adv Neurol. 1990;53:17-29.

54. Graybiel AM. Neurotransmitters and neuromodulators in the basal ganglia. Trends Neurosci. 1990;13(7):244-54.

55. Canales JJ, Graybiel AM. Patterns of gene expression and behavior induced by chronic dopamine treatments. Ann Neurol. 2000;47(4 Suppl 1):S53-9.

56. Bloem B, Huda R, Sur M, Graybiel AM. Two-photon imaging in mice shows striosomes and matrix have overlapping but differential reinforcement-related responses. Elife. 2017;6:e32353.
57. Ostlund SB, Wassum KM, Murphy NP, Balleine BW, Maidment NT. Extracellular dopamine levels in striatal subregions track shifts in motivation and response cost during instrumental conditioning. J Neurosci. 2011;31(1):200-7.

58. Yin HH, Knowlton BJ, Balleine BW. Lesions of dorsolateral striatum preserve outcome expectancy but disrupt habit formation in instrumental learning. Eur J Neurosci. 2004;19(1):181-9.

59. Yin $\mathrm{HH}$, Knowlton BJ, Balleine BW. Inactivation of dorsolateral striatum enhances sensitivity to changes in the action-outcome contingency in instrumental conditioning. Behav Brain Res. 2006;166(2):189-96.

60. Yin HH, Ostlund SB, Knowlton BJ, Balleine BW. The role of the dorsomedial striatum in instrumental conditioning. Eur J Neurosci. 2005;22(2):513-23.

61. Cragg SJ. Variable dopamine release probability and short-term plasticity between functional domains of the primate striatum. J Neurosci. 2003;23(10):4378-85.

62. Gerfen C, Herkenham M, Thibault J. The neostriatal mosaic: II. Patch- and matrix- directed mesostriatal dopaminergic and nondopaminergic systems. J Neurosci. 1987;7(12):3915-34.

63. Gerfen CR. The neostriatal mosaic: compartmentalization of corticostriatal input and striatonigral output systems. Nature. 1984;311(5985):461-4.

64. Brimblecombe KR, Cragg SJ. The striosome and matrix compartments of the striatum: a path through the labyrinth from neurochemistry toward function. ACS Chem Neurosci. 2017;8(2):235-42.

65. Shu Z, Taylor IM, Walters SH, Michael AC. Region- and domaindependent action of nomifensine. Eur J Neurosci. 2014;40(2): 2320-8.

66. Walters SH, Robbins EM, Michael AC. Modeling the kinetic diversity of dopamine in the dorsal striatum. ACS Chem Neurosci. 2015;6(8):1468-75.

67. Walters SH, Taylor IM, Shu Z, Michael AC. A novel restricted diffusion model of evoked dopamine. ACS Chem Neurosci. 2014;5(9):776-783.

68. Brimblecombe KR, Cragg SJ. Substance P weights striatal dopamine transmission differently within the striosome-matrix axis. J Neurosci. 2015;35(24):9017-23.

69. Salinas AG, Davis MI, Lovinger DM, Mateo Y. Dopamine dynamics and cocaine sensitivity differ between striosome and matrix compartments of the striatum. Neuropharmacology. 2016;108: 275-83.

70. Schmitz Y, Benoit-Marand M, Gonon F, Sulzer D. Presynaptic regulation of dopaminergic neurotransmission. J Neurochem. 2003;87(2):273-89.

71. Brimblecombe KR, Threlfell S, Dautan D, Kosillo P, MenaSegovia J, Cragg SJ. Targeted activation of cholinergic interneurons accounts for the modulation of dopamine by striatal nicotinic receptors. eNeuro. 2018;5(5):ENEURO.0397-17.2018.

72. Sulzer D. How addictive drugs disrupt presynaptic dopamine neurotransmission. Neuron. 2011;69(4):628-49.

73. Zhang H, Sulzer D. Frequency-dependent modulation of dopamine release by nicotine. Nat Neurosci. 2004;7(6):581-2.

74. Zhang S, Qi J, Li X, Wang HL, Britt JP, Hoffman AF, et al. Dopaminergic and glutamatergic microdomains in a subset of rodent mesoaccumbens axons. Nat Neurosci. 2015;18(3):386-92.

Publisher's note Springer Nature remains neutral with regard to jurisdictional claims in published maps and institutional affiliations. 


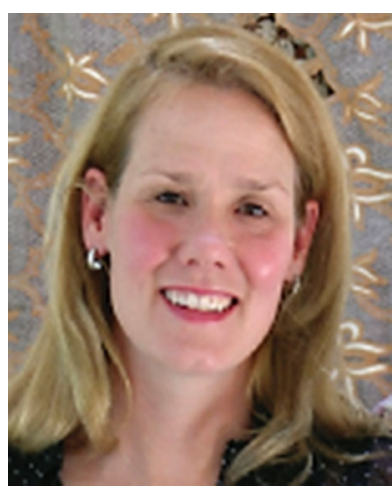

Andrea Jaquins-Gerstl received her Ph.D. in chemistry from Carnegie Mellon University, Pittsburgh, PA, in 2007 where she worked on single molecule spectroscopy using quantum dots/gold nanoparticles and biosensors. Later that year, she joined Adrian Michael's group at the University of Pittsburgh, Pittsburgh, PA. Currently, she is Assistant Research Professor of Chemistry at the University of Pittsburgh and works on mitigating tissue damage caused by microdialysis and voltammetry insertions in the brain.

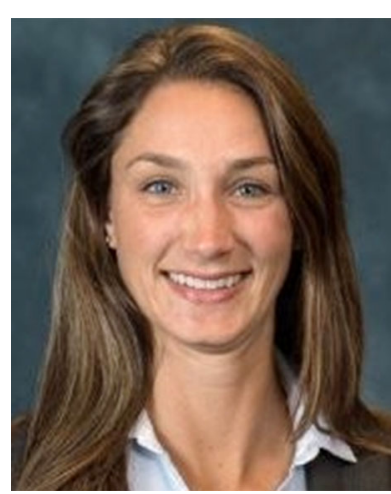

Kathryn Nesbitt is former Assistant Professor of Chemistry at Towson University. She received her Ph.D. in chemistry from the University of Pittsburgh in 2015 where she worked on mitigating tissue damage during microdialysis. Following her Ph.D., she spent 2 years as a post-doc at the University of Michigan analyzing the effects of obesity and diet on neurotransmitters.

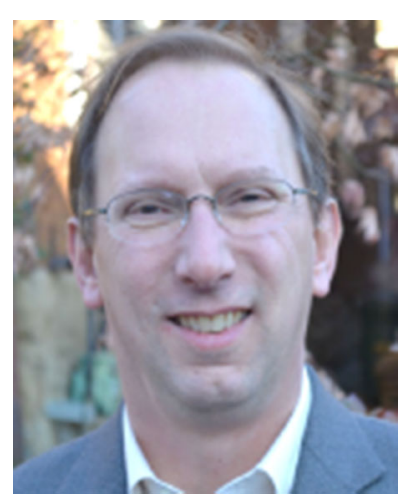

Adrian C. Michael received his Ph.D. in chemistry from Emory University, Atlanta, GA, in 1987. He spent 2 years at Indiana University, Bloomington, IN, and 1 year at the University of Texas at Austin, TX, before moving to Pittsburgh, PA. Currently, he is Professor of Chemistry at the University of Pittsburgh, Pittsburgh, PA. His research focuses on monitoring DAergic processes in living animals using electro-analytical techniques in conjunction with microelectrodes and microdialysis probes. Penetration injury associated with microdialysis and voltammetry, mitigating tissue damage during microdialysis, and dynamic dopamine processes in the brain are included in his research. 